Journal of Clinical Endocrinology and Metabolism (DOI: 10.1210/clinem/dgz119)

\title{
Elevated cholesteryl ester transfer protein activity early in pregnancy predicts prediabetes 5
} years later

Thor Ueland, $\mathrm{PhD}^{1,2,3}$, Marie Cecilie Paasche Roland, $\mathrm{MD}, \mathrm{PhD}^{4,5}$, Annika E. Michelsen, $\mathrm{PhD}^{1,2}$, Kristin

Godang, BSc ${ }^{6}$, Pål Aukrust, $\mathrm{MD}, \mathrm{PhD}^{1,2,3,7,8}$, Tore Henriksen, $\mathrm{MD}, \mathrm{PhD}^{2,5}$, Jens Bollerslev, $\mathrm{MD}$, $\mathrm{DMSc}^{2,6}$, Tove Lekva, $\mathrm{PhD}^{1}$

${ }^{1}$ Research Institute of Internal Medicine, Oslo University Hospital, Rikshospitalet, Oslo, Norway ${ }^{2}$ Faculty of Medicine; University of Oslo, Oslo, Norway

${ }^{3}$ K. G. Jebsen Thrombosis Research and Expertise Center, University of Tromsø, Tromsø, Norway ${ }^{4}$ National Advisory Unit for Womens Health; Oslo University Hospital, Rikshospitalet, Oslo, Norway

${ }^{5}$ Department of Obstetrics, Oslo University Hospital, Rikshospitalet, Oslo, Norway

${ }^{6}$ Section of Specialized Endocrinology, Department of Endocrinology, Oslo University Hospital, Rikshospitalet, Oslo, Norway

${ }^{7}$ Section of Clinical Immunology and Infectious Diseases, Oslo University Hospital, Rikshospitalet, Oslo, Norway

${ }^{8}$ K.G. Jebsen Inflammatory Research Center, University of Oslo, Oslo, Norway

\section{Tables 6, Figures 3}

word count manuscript: 3490 word count abstract: 229

Short title: CETP activity in pregnancy predicts prediabetes

Keywords; Prediabetes, CETP activity

\section{Corresponding author:}

Tove Lekva, $\mathrm{PhD}$

Research Institute of Internal Medicine, Oslo University Hospital, Rikshospitalet, Oslo, Norway

E-mail: tove.lekva@,rr-research.no 


\section{Funding}

35 The project has been financially supported by grants from Diabetesforbundet (TL) and Anders Jahres Foundation (TL). The funders had no role in study design, data collection and analysis, decision to publish, or preparation of the manuscript.

38 
Abstract

Context: Cholesteryl ester transfer protein (CETP) regulates high density lipoproteins (HDL)-

cholesterol levels and interaction between glucose and HDL metabolism is central in the development of diabetes.

Objective: We hypothesized that CETP levels would be regulated in diabetic pregnancies. We tested the hypothesis by evaluating CETP activity measured multiple times during pregnancy and at 5 years follow-up in a prospective cohort (STORK) and investigated its association with gestational diabetes mellitus (GDM) during pregnancy or development of prediabetes 5 years after pregnancy. We also evaluated the strongest correlated of CETP activity among measures of adipocity and glucose metabolism, lipoproteins, adipokines and monocyte/macrophage activation markers.

Design: Population-based longitudinal cohort study from 2001 to 2013.

Setting: Oslo University Hospital.

Patients or other Participants: 300 women during pregnancy and at 5 years postpartum.

Main Outcome Measures: CETP activity measured at 14-16, 22-24, 30-32, 36-38 weeks gestation, and at 5 years follow-up.

Results: We found higher CETP activity in pregnancy in women developing prediabetes but no association with GDM. CETP activity decreased throughout pregnancy and remained low at followup. High CETP activity was associated with sCD14 levels, in particular in women who developed prediabetes. These data show that enhanced CETP activity during pregnancy is associated with systemic indices of monocyte/macrophage activation, in particular in women who develop prediabetes later in life.

Conclusions: CETP activity during pregnancy identified women at risk for later diabetes development. 
Precis: Our study shows that CETP activity during pregnancy identified women at risk for later

87 diabetes development.

88

\section{Abbreviations}

90

91 CETP Cholesteryl ester transfer protein

92 GDM Gestational diabetes mellitus

93 HDL High density lipoproteins

94 LDL Low density lipoproteins

95 OGTT Oral glucose tolerance test

96 TG Triglycerides

97 VLDL Very low density lipoprotein

98

99

100

101

102

103

104

105

106

107

108

109

110

111

112

113 


\section{Introduction}

Both the number and composition of lipoprotein particles change during pregnancy. Early pregnancy is characterized by increased hepatic production and systemic removal of triglycerides (TG), with deposition of fat in maternal adipose tissue. Later, TG levels rise markedly, and high levels of TGenriched lipoproteins are observed mainly due to estrogen-driven hepatic synthesis of very low density lipoprotein (VLDL) and attenuated removal of TG. The abundance of VLDL TG accelerates transfer of TG to lipoproteins of higher density by cholesteryl ester transfer protein (CETP). Thus, through enhanced CETP activity high density lipoprotein (HDL) becomes progressively poorer in cholesterol ester and richer in TG (1). pro-atherogenic non-HDL fractions (ApoB, LDL, VLDL and IDL) in exchange for TG $(2,3)$. Increased CETP activity may increase TG in the HDL core, resulting on lower plasma HDL cholesterol (HDL-C) with potentially pro-atherogenic net effects. Elevated CETP activity has been observed in insulin resistance conditions, like obesity and type 2 diabetes (4). Inhibition of CETP substantially increases HDL-C and reduces non-HDL-C levels (2). A recent meta-analysis indicated that CETP inhibitor therapy significantly (12\%) reduced the incidence of diabetes (5). Large clinical studies using CETP inhibition therapy investigating cardiovascular outcome showed only modest improvements in prognosis, although a significant reduction in cardiovascular events was observed in the REVEAL study in combination with statins, and the beneficial effect also improved glycaemic control (6).

Low HDL-C is associated with insulin resistance and development of diabetes (7). It is not known whether a low HDL level is causal, but there is evidence that HDLs enhances insulin synthesis, secretion and has anti-apoptotic effect on pancreatic beta-cells $(8,9)$. Further, HDLs have anti-diabetic effects in adipose tissue and enhance glucose uptake by skeletal muscle (3). Low HDL-C is observed in gestational diabetes mellitus (GDM) (10) and during long-term follow-up after a GDM diagnosis 
142 (11). Prediabetes is shown to have an increased risk, in addition to type 2 diabetes, of cardiovascular 143 disease and all-cause mortality (12) giving this group a high priority in optimizing glycemic control.

Based on the role of CETP in regulating HDL-C levels and interaction between glucose and HDL metabolism in the development of diabetes, we hypothesized that CETP levels would be regulated in diabetic pregnancies. We therefore evaluated CETP activity measured multiple times during pregnancy and at 5 years follow-up in a prospective cohort (STORK) of 300 women and investigated its association with i) GDM during pregnancy and the development of prediabetes 5 years after pregnancy, and ii) measures of adipocity and glucose metabolism, lipoproteins, adipokines and monocyte/macrophage activation markers.

\section{Material and Methods}

The STORK study, a prospective longitudinal cohort study in which 1031 low-risk women of Scandinavian heritage were followed throughout their pregnancy and gave birth at Oslo University Hospital, Rikshospitalet between 2002 and 2008 (13). The exclusion criteria were multiple pregnancies, known pre-gestational diabetes and any severe chronic diseases (lung, cardiac, gastrointestinal or renal). Each pregnant woman had four study-related antenatal visits at weeks 14-16, 22-24, 30-32, and 36-38. A 75g OGTT was performed in all women at 14-16 and again at 30-32 weeks of gestation. All women were invited to participate in a 5-year postpartum follow-up study of whom 300 from the original study agreed (14). We included only the women who had participated both during pregnancy and follow-up in this particular study. Women with preeclampsia were excluded, and this study ended up with 290 participants. Subcutaneous fat at the triceps, subscapular, and iliac sites were estimated during pregnancy using a Holtain caliper (Holtain, Crymych, UK). Written informed consent was obtained from all study participants. All clinical investigations were conducted in accordance with the principles enshrined in the Declaration of Helsinki. The study was approved by the Regional Committee for Medical Research Ethics of Southern Norway in Oslo, Norway.

Measurements of glucose and insulin from OGTT 
All 75g OGTTs were performed in the morning after an overnight fast and glucose levels measured as previously reported (14). Briefly, venous blood was drawn in gel tubes, allowed to clot for $30 \mathrm{~min}$, thereafter centrifuged for $10 \mathrm{~min} 3000 \mathrm{~g}$, serum separated and stored at $-80^{\circ} \mathrm{C}$. Glucose was measured in serum samples collected at antenatal visits at $14-16$ and 30-32 weeks and frozen until analysis, using the hexokinase method at an accredited clinical chemistry laboratory at Oslo University Hospital (Cobas 6000 from Roche). For the 5-year follow-up visit, fasting glucose measurements were collected using an Accu-check Sensor glucometer (Roche Diagnostics), using venous EDTA blood analyzed on site, as previously reported (14). Insulin levels were assayed in duplicate (RIA, DPC, Los Angeles, CA, USA) as previously reported $(14,15)$.

Diagnosis of GDM and pre-diabetes

GDM was diagnosed using the WHO2013 criteria (fasting plasma glucose (FPG) $5.1-6.9 \mathrm{mmol} / \mathrm{L}, 1 \mathrm{~h}$ plasma glucose $\geq 10.0 \mathrm{mmol} / \mathrm{L}$ or $2 \mathrm{~h}$ plasma glucose $8.5-11.0 \mathrm{mmol} / \mathrm{L}$ ), at any time in pregnancy following a $75 \mathrm{~g}$ oral glucose load. Pre-diabetes was diagnosed at the 5-year follow-up visit using the following criteria: FPG 5.6-6.9mmol/L or $2 \mathrm{~h}$ plasma glucose $7.8-11.0 \mathrm{mmol} / \mathrm{L}$ after $75 \mathrm{~g}$ OGTT (16).

Insulin sensitivity was measured with the Matsuda index 10000/ $\sqrt{ }$ of (fasting glucose $(\mathrm{mmol} / \mathrm{L}) \times$ fasting insulin $(\mathrm{mU} / \mathrm{L}) \times($ mean glucose $(\mathrm{mmol} / \mathrm{L}) \times$ mean insulin $(\mathrm{mU} / \mathrm{L}))$ during $75 \mathrm{~g}$ OGTT . This index is a measure of whole body insulin sensitivity that has been validated against the euglycemic-hyperinsulinemic clamp (17). $\beta$-cell function was assessed with the insulin secretionsensitivity index (ISSI-2) (area under the curve insulin(mU/L $)_{0-120} /$ glucose $(\mathrm{mmol} / \mathrm{L})_{0-120} \times$ Matsuda), validated against the disposition index from the intravenous glucose tolerance test (18). HOMA-IR was calculated as fasting insulin $(\mathrm{mU} / \mathrm{L}) \times$ fasting glucose $(\mathrm{mmol} / \mathrm{L}) / 22.5$, as described by Matthews et al. (19).

\section{Lipoproteins and lipids}

Lipoproteins and lipids were measured at an accredited laboratory at Oslo University Hospital, Rikshospitalet. Total cholesterol, HDL-C and triglycerides were measured at weeks 14-16 and 36-38 during pregnancy, as previously reported (Roland et al, under review). LDL-C was determined by 
Friedewald's formula (20). Levels of HDL-C, LDL-C (directly measurements), and triglycerides (TG) were measured at follow-up as previously reported (11).

Measurement of adipokines, monocyte/macrophage and inflammatory markers

Peripheral venous blood was drawn into pyrogen-free tubes with EDTA as anticoagulant. The tubes were immediately immersed in melting ice and centrifuged within 30 minutes at 2,000g for 20 minutes to obtain platelet-poor plasma. All samples were stored at $-80^{\circ} \mathrm{C}$ and thawed $<3$ times. Adipokines (adiponectin (DY1065), chemerin (DY2324), leptin (DY398), resistin (DY1359)), monocyte/macrophage markers (sCD163 (DY1607), sCD14 (DY383)) and the inflammatory marker CRP (DY1707) were measured in duplicate using commercially available antibodies (R\&D Systems, Minneapolis, MN, USA) as previously reported (21-23) using a 384 format using the combination of a SELMA (Jena, Germany) pipetting robot and a BioTek (Winooski, VT, USA) dispenser/washer (EL406). Absorption was read at $450 \mathrm{~nm}$ with wavelength correction set to $540 \mathrm{~nm}$ using an ELISA plate reader (Synergy H1 Hybrid, Biotek, Vinooski, VT, USA). Intra- and inter-assay coefficients of variation were $<10 \%$ for all assays.

\section{Measurement of CETP activity}

Plasma CETP activity was measured in duplicate using commercially available kit (MAK106) from Sigma-Aldrich (St. Louis, MO). The reaction mixture contained a donor molecule that was a fluorescent self-quenching neutral lipid as well as an acceptor molecule. Five $\mu \mathrm{L}$ of diluted plasma sample was added to the reaction mixture and incubated for 3 hours at $37^{\circ} \mathrm{C}$ in a black 384 well plate. CETP-mediated transfer from donor to acceptor resulted in an increase in fluorescence intensity with an excitation wavelength of $465 \mathrm{~nm}$ and emission of $535 \mathrm{~nm}$ as read by the fluorescent plate reader. The CV for the analysis was $<13 \%$. All 5 samples from one person were analyzed on the same plate.

Statistical analysis

Statistical analyses were conducted using SPSS for Windows, version 21.0. Data are expressed as mean (SD) when normally distributed and median $\left(25^{\text {th }}, 75^{\text {th }}\right.$ percentile $)$ when skewed. 
For comparison of demographic and baseline data between prediabetes women vs. non-diabetes women, or GDM vs. non-GDM, students $t$-test or Mann-Whitney's $U$ test were used depending on distribution, and $\chi^{2}$ test for categorical variables (Table 1 and 2). Temporal changes in CETP activity, lipoproteins, and inflammatory markers were assessed with repeated measures ANOVA, and if the group effect was significant, multivariate linear regression analyses were carried out on log transformed variables (if skewed) at each visit, adjusting for age and BMI. These data are reported as back-transformed estimated marginal means with 95\% confidence intervals (Figure 1, Figure 2 and Table 3). For evaluating predictors of CETP values at each individual time-point, we used stepwise linear regression (Table 4). To identify the strongest predictors of prediabetes and $2 \mathrm{~h}$ glucose during OGTT using logistic and linear regression, respectively, we first performed univariate analysis and included all variables with $\mathrm{p}<0.2$ in the adjusted multivariable analysis (Table 5). In addition, the modifying effect of each confounding variable in Table 5 on the association between CETP and prediabetes and 2-h glucose at 5 years follow-up was assessed using linear and logistic regression (Table 6). Spearman correlation was used when analyzing correlation between CETP activity and sCD14 at different time-points (Figure 3). Interaction analysis on 2-hour glucose levels as dependent and CETP and sCD14 at 14-16 weeks as independent was performed with both proteins and their product (Figure 3E). To visualize this we divided CETP and sCD14 in tertiles and graphed their product in relation to 2-hour glucose levels. This gave 6 groups (Figure 3F): group 1: Tertile 1 (T1) of both CETP and sCD14; group 2: combinations of T1 and T2 of CETP and sCD14; group 3: combinations of T1 and T3 of CETP and sCD14; group 4: T2 of both; group 5: combinations of T2 and T3 of CETP and sCD14; group 6: T3 of both. P-values are two-sided and $\mathrm{p}<0.05$ was considered statistically significant.

\section{Results}

Table 1 shows the characteristics of the prediabetes population (i.e. FPG 5.6-6.9mmol/L or $2 \mathrm{~h}$ plasma glucose $7.8-11.0 \mathrm{mmol} / \mathrm{L}$ after $75 \mathrm{~g}$ OGTT) vs. the non-diabetes population at 5 years follow-up and retrospective during pregnancy. Table 2 shows the characteristics of the GDM diagnosed with the 
WHO 2013 diagnostic criteria vs. non-GDM women at the same timepoints. Briefly, GDM women were older and had a higher BMI and weight than controls while the prediabetes women only had

250

251

252 higher weight during pregnancy and elevated BMI at 5 years follow-up. Systolic blood pressure was higher in GDM while diastolic blood pressure was higher in prediabetes. Indices of glucose metabolism were dysregulated in both the GDM and prediabetes group.

\section{Lipoproteins and lipids in GDM and prediabetes}

We have previously presented lipoprotein levels (Roland et al, submitted) and cardio-metabolic lipid ratios during pregnancy and at 5 years follow-up (11). In the present study we found a similar lipoprotein dysregulation in prediabetes as in GDM characterized by low HDL-C at week 14-16, and low HDL-C, high TG and TG/HDL ratio at 5 years follow-up (Figure 1).

\section{CETP activity in GDM and women who develop prediabetes}

Evaluating women identified with prediabetes at 5 years follow-up $(n=20)$ during pregnancy revealed elevated levels of CETP activity at 14-16 weeks, 22-24 weeks, 30-32 weeks and 36-38 weeks. Of these prediabetes women at 5 years follow-up, 14 were diagnosed with GDM and 6 were not diagnosed with GDM during pregnancy (Figure 2). We found no difference in CETP activity between GDM ( $\mathrm{n}=70)$ and non-GDM $(\mathrm{n}=215)$ at any time point during pregnancy or at follow-up.

Preeclampsia patients were excluded from the analysis.

CETP activity associations with clinical markers, glucose tolerance, lipoproteins, inflammatory markers, monocyte/macrophage markers and adipokines

We next evaluated predictors of CETP levels at 14-16 weeks, 30-32 weeks and at 5 years follow-up using variables obtained at the same time-point. As seen in Table 4, CETP activity correlated with subcutaneous fat in the iliaca region, but not triceps or BMI. Further, CETP was modestly associated with indices of glucose metabolism. For lipoproteins, a positive association with LDL-C at 14-16 weeks, at term and follow-up was observed while a negative correlation with HDL-C was seen at 1416 weeks, and a negative association with TG at term. 
We have previously measured adipokines, monocyte/macrophage and inflammatory markers

in this cohort (22). As seen in Table 4, leptin, resistin, chemerin, sCD163, sCD14, CRP were positively correlated with CETP activity during pregnancy. Soluble CD163 and sCD14 were also associated with CETP activity at 5 years follow-up. Multivariable linear regression, revealed sCD14 as the strongest determinant of CETP activity during and after pregnancy. Levels of these markers in women who developed prediabetes and non-diabetes are shown in Table 3.

\section{Association between CETP activity and sCD14 during pregnancy}

As described above, sCD14 was consistently one of the strongest predictors of CETP activity. Figure 3A shows CETP activity and sCD14 levels at 14-16 weeks as well as the AUC for these markers during pregnancy. A similar pattern of CETP activity and sCD14 was observed across the different groups of normal pregnancy, GDM and prediabetes. Figure $3 \mathrm{C}$ shows the correlation between AUCs for sCD14 and CETP activity indicating a stronger correlation in women who became prediabetic at follow-up. This correlations was evident at all time-points during pregnancy (Figure 3D) but lacking in GDM women. Finally, regression analysis of sCD14 and CETP at 14-16 weeks as predictors of 2-hour glucose at follow-up reveled an interaction (Figure 3E). To visualize this we graphed the product of tertiles of both proteins giving six groups (See statistical methods and Figure 3F). As shown in Figure 3F, the 3 first groups had similar 2-hour glucose levels, including group 3 which consisted of combinations of tertile 1 and 3 of CETP or sCD14, indicating that having high levels of CETP alone is not associated with future prediabetes. However combinations of tertile 2 and 3 of CETP and sCD14 had higher levels of 2-hour glucose than the other groups indicating that high levels of both are more strongly associated with prediabetes.

\section{Elevated CETP activity early in pregnancy predicts prediabetes 5 years after pregnancy}

Logistic regression investigating the strongest early predictors of prediabetes (i.e. at week 14-16) found CETP (OR [CI]: 2.13 [1.18-3.81], $\mathrm{p}=0.012)$ and $\beta$-cell function $(0.35$ [0.19-0.66] $\mathrm{p}=0.001)$ as predictors of prediabetes at 5 years follow-up. Using linear regression and evaluating glucose tolerance as a continuous measure identified $\beta$-cell function (Slope -0.22 95\% CI (-0.34- -0.10), 
$\mathrm{p}<0.001)$ and CETP (Slope $0.15(0.03-0.27), \mathrm{p}=0.012)$ at week $14-16$ as the strongest predictors of 2 hours glucose during OGTT at 5 years follow-up (Table 5).

302

303

\section{Discussion}

Our prospective cohort study evaluating CETP activity during pregnancy in normal and GDM women and in prediabetes 5 years follow-up revealed i) higher CETP activity in pregnancy in women developing prediabetes but no association with GDM ii) CETP activity decreased throughout pregnancy and remained low at follow-up iii) high CETP activity was associated with sCD14 levels, in particular in women who developed prediabetes and iv) CETP measurements during pregnancy was an independent and strong predictor of developing prediabetes 5 years after delivery. These data show that enhanced CETP activity during pregnancy is associated with development of prediabetes after pregnancy, but not with GDM, potentially involving interaction with monocyte/macrophage activation.

The protective role of HDL has been ascribed to its capacity to promote reverse cholesterol transport from peripheral cells and deliver it to the liver for excretion. CETP is an important factor in HDL metabolism and reciprocal changes in CETP activity and HDL plasma levels are well documented (1). Elevated CETP activity during second trimester has been reported in normolipidemic healthy women, coinciding with the greatest increase in LDL- and HDL TG content, with similar changes during pregnancy and postpartum as shown in the present study $(1,24)$. While CETP was negatively associated with HDL activity early in pregnancy and positively with LDL during pregnancy and at follow-up, these correlations were modest in the present study. Instead, the monocyte/macrophage markers sCD163 and in particular sCD14, were positively associated with CETP activity both during pregnancy and at follow-up, supporting a link between monocyte/macrophage activation and CETP activity. Plasma CETP levels have previously been shown to correlate with liver macrophage content and it has been suggested that hepatic macrophages are the main producer of plasma CETP (25). Moreover, large increase in macrophage content in skeletal muscle and increase in CETP plasma levels after high fat diet has been documented (26). Obesity is associated with a lower HDL-mediated cholesterol efflux from macrophages and higher CETP activity 
(27). It is therefore possible that CETP could be an important mediator linking HDL-C and inflammation with macrophages as a crucial cell. Based on our data, these mechanisms could potentially also contribute to the development of prediabetes following pregnancy.

The main finding of our study was that CETP activity during pregnancy identified women at risk for later diabetes development, regardless of GDM status, which to our knowledge has not previously been reported. Furthermore, the association between sCD14 and CETP activity was much stronger in women who developed prediabetes compared to both women with uncomplicated pregnancy and GDM. Although sCD14 levels were not significantly different between women with normal pregnancy and those who developed prediabetes, there was a uniform trend of higher sCD14 levels in prediabetic women and stronger correlation between SCD14 and CETP activity at all-timepoints during pregnancy. Furthermore, of all variables including measures of adipocity, lipoproteins, indices of glucose metabolism and inflammatory markers, sCD14 was consistently identified as a strong predictor of CETP both during pregnancy and at 5-year follow-up. Furthermore, the statistical interaction between CETP and SCD14 at 14-16 weeks in predicting 2-hour glucose further supports a link between CETP activity, monocyte/macrophage activation and diabetes. Although sCD163 also is a monocyte/macrophage activation marker, the lack of regulation in women who develop prediabetes could suggest that $\mathrm{sCD} 14$ is reflecting M1 activation since sCD163 is considered a M2 marker (28). Indeed, enhanced activation of CD14+ monocytes with increased ability for endothelial cell attachment has been described in diabetes (29) and polarization towards a pro-inflammatory M1 phenotype has been demonstrated in prediabetes (30). Monocyte/macrophage activation occurs during normal pregnancy and although the precise mechanisms are unknown, exposure of maternal blood to placental cells or inflammatory products from these may activate them towards a pro-inflammatory phenotype (31). During LPS/TLR4 interaction sCD14 is released from monocytes/macrophages and notably, LPS from gut microbiota could interact with monocytes/macrophages outside the intestine through gut leakage mechanisms into the systemic circulation, which could be particular relevant during pregnancy (32). We recently demonstrated enhanced sCD163 in early GDM pregnancies with an inverse association with $\beta$-cell function, particularly in women with high BMI (22). 
In the present study, $\beta$-cell function and CETP activity at $14-16$ weeks gestation were the strongest predictors of prediabetes and the $2 \mathrm{~h}$ OGTT glucose at 5 years follow-up. Thus, based on the strong correlation between sCD14 and CETP in women who developed prediabetes, our study may reflect the importance of monocyte/macrophage activation in the initiation of diabetic complications, possibly involving CETP related mechanisms. However, within the prediabetes group, the correlation between CETP activity and SCD14 was not present post-partum indicating that interactions between monocyte/macrophage activation and CETP may be more dominant during metabolic stress as in pregnancy.

An interaction between monocyte/macrophage activation and CETP activity may also be relevant for adipose tissue as cross-talk between these cells may influence obesity associated insulin resistance as well as progression of atherosclerosis (33). Increased CD14 content in epicardial adipose tissue has been demonstrated in type 2 diabetes (34). In addition to reflecting monocyte/macrophage activation, CD14 may also directly modulate adipose tissue inflammatory activity and insulin resistance (35). CETP is also expressed in human adipose tissue (36). Plasma CETP is positively correlated with its mRNA expression in pericardial fat (37), and overexpression of CETP in mouse adipose tissue elevates plasma CETP (38). These findings suggest that adipose tissue contributes to plasma levels of this lipid transfer protein. In our study, we detected no correlation between CETP activity and BMI. However, CETP activity correlated with subcutaneous fat iliaca measured by caliper as well as several adipose tissue markers linked to diabetes progression suggesting that regional fat distribution could influence CETP activity. Indeed, CETP is predominantly expressed in subcutaneous adipose tissue compared to visceral adipose tissue (39). Furthermore, these associations were only present during pregnancy and not at follow-up suggesting an association between adipose tissue accumulation and CETP activity.

Our study has several limitations. It is an observational study, and thus, we cannot explain the mechanisms underlying the findings. The low number of prediabetes at 5 years follow-up is the main weakness. However, our cohort is population based and we have not identified selection bias. In addition, the women in the study were young and early development of diabetes is important to 
381 investigate on the way to discover alternative actions to delay the disease to further progress. The lack

382 of associations at follow-up might thus be because the women are young and the metabolic

383 complications have not been manifested yet. We could speculate that a longer follow-up might reveal

384 increased levels of CETP in both the prediabetes group and in the women in the GDM group who are

385 prone to develop type 2 diabetes. Further, more mechanistic and larger studies should replicate these

386 findings.

387 In conclusion, CETP activity was only modestly associated with HDL metabolism during

388 pregnancy, but was more closely associated with sCD14 reflecting monocyte/macrophage activation.

389 CETP activity during pregnancy identified women at risk for later diabetes development. Clearly,

390 further investigations into the link between CETP activity, monocyte/macrophage activation and

391 diabetes are warranted including experimental studies evaluating how these interactions are modified

392 by CETP inhibition. If successful, such studies reveal novel treatment strategies to prevent

393 development of diabetes associated with pregnancy.

394

395

396

397

398

399

400

401

402

403

404

405

406

407

408

409 
1. Iglesias A, Montelongo A, Herrera E, Lasuncion MA. Changes in cholesteryl ester transfer protein activity during normal gestation and postpartum. Clin Biochem. 1994;27:63-68.

2. Group HTRC, Bowman L, Hopewell JC, Chen F, Wallendszus K, Stevens W, Collins R, Wiviott $\mathrm{SD}$, Cannon CP, Braunwald E, Sammons E, Landray MJ. Effects of anacetrapib in patients with atherosclerotic vascular disease. N Engl J Med. 2017;377:1217-1227.

3. Barter PJ, Cochran BJ, Rye KA. Cetp inhibition, statins and diabetes. Atherosclerosis. 2018;278:143-146.

4. Borggreve SE, De Vries R, Dullaart RP. Alterations in high-density lipoprotein metabolism and reverse cholesterol transport in insulin resistance and type 2 diabetes mellitus: Role of lipolytic enzymes, lecithin:Cholesterol acyltransferase and lipid transfer proteins. Eur J Clin Invest. 2003;33:1051-1069.

5. Masson W, Lobo M, Siniawski D, Huerin M, Molinero G, Valero R, Nogueira JP. Therapy with cholesteryl ester transfer protein (cetp) inhibitors and diabetes risk. Diabetes Metab. 2018;44:508-513.

6. Armitage J, Holmes MV, Preiss D. Cholesteryl ester transfer protein inhibition for preventing cardiovascular events: Jacc review topic of the week. J Am Coll Cardiol. 2019;73:477-487.

7. Montonen J, Drogan D, Joost HG, Boeing H, Fritsche A, Schleicher E, Schulze MB, Pischon T. Estimation of the contribution of biomarkers of different metabolic pathways to risk of type 2 diabetes. Eur J Epidemiol. 2011;26:29-38.

8. von Eckardstein A, Widmann C. High-density lipoprotein, beta cells, and diabetes. Cardiovasc Res. 2014;103:384-394.

9. Siebel AL, Natoli AK, Yap FY, Carey AL, Reddy-Luthmoodoo M, Sviridov D, Weber Cl, MenesesLorente G, Maugeais C, Forbes JM, Kingwell BA. Effects of high-density lipoprotein elevation with cholesteryl ester transfer protein inhibition on insulin secretion. Circ Res. 2013;113:167175.

10. Ryckman KK, Spracklen CN, Smith CJ, Robinson JG, Saftlas AF. Maternal lipid levels during pregnancy and gestational diabetes: A systematic review and meta-analysis. BJOG. 2015;122:643-651.

11. Lekva T, Bollerslev J, Norwitz ER, Aukrust P, Henriksen T, Ueland T. Aortic stiffness and cardiovascular risk in women with previous gestational diabetes mellitus. PLOS.One. 2015;10:e0136892.

12. Huang Y, Cai X, Mai W, Li M, Hu Y. Association between prediabetes and risk of cardiovascular disease and all cause mortality: Systematic review and meta-analysis. BMJ. 2016;355:i5953.

13. Roland MC, Friis CM, Voldner N, Godang K, Bollerslev J, Haugen G, Henriksen T. Fetal growth versus birthweight: The role of placenta versus other determinants. PLOS.One. 2012;7:e39324.

14. Lekva T, Bollerslev J, Godang K, Roland MC, Friis CM, Voldner N, Henriksen T, Ueland T. Betacell dysfunction in women with previous gestational diabetes is associated with visceral adipose tissue distribution. Eur.J.Endocrinol. 2015;173:63-70. 
15. Qvigstad E, Voldner N, Godang K, Henriksen T, Bollerslev J. Overweight is associated with impaired beta-cell function during pregnancy: A longitudinal study of 553 normal pregnancies. Eur.J.Endocrinol. 2010;162:67-73.

16. Standards of medical care in diabetes--2013. Diabetes Care. 2013;36 Suppl 1:S11-S66.

17. Matsuda $M$, DeFronzo RA. Insulin sensitivity indices obtained from oral glucose tolerance testing: Comparison with the euglycemic insulin clamp. Diabetes Care. 1999;22:1462-1470.

18. Retnakaran R, Shen S, Hanley AJ, Vuksan V, Hamilton JK, Zinman B. Hyperbolic relationship between insulin secretion and sensitivity on oral glucose tolerance test.

Obesity.(Silver.Spring). 2008;16:1901-1907.

19. Matthews DR, Hosker JP, Rudenski AS, Naylor BA, Treacher DF, Turner RC. Homeostasis model assessment: Insulin resistance and beta-cell function from fasting plasma glucose and insulin concentrations in man. Diabetologia. 1985;28:412-419.

20. Friedewald WT, Levy RI, Fredrickson DS. Estimation of the concentration of low-density lipoprotein cholesterol in plasma, without use of the preparative ultracentrifuge. Clin Chem. 1972;18:499-502.

21. Lekva T, Michelsen AE, Bollerslev J, Norwitz ER, Aukrust P, Henriksen T, Ueland T. Low circulating pentraxin 3 levels in pregnancy is associated with gestational diabetes and increased apob/apoa ratio: A 5-year follow-up study. Cardiovasc Diabetol. 2016;15:23.

22. Ueland T, Michelsen AE, Aukrust P, Henriksen T, Bollerslev J, Lekva T. Adipokines and macrophage markers during pregnancy-possible role for scd163 in prediction and progression of gestational diabetes mellitus. Diabetes Metab Res Rev. 2019;35:e3114.

23. Lekva T, Michelsen AE, Aukrust P, Henriksen T, Bollerslev J, Ueland T. Leptin and adiponectin as predictors of cardiovascular risk after gestational diabetes mellitus. Cardiovasc Diabetol. 2017;16:5.

24. Alvarez JJ, Montelongo A, Iglesias A, Lasuncion MA, Herrera E. Longitudinal study on lipoprotein profile, high density lipoprotein subclass, and postheparin lipases during gestation in women. J Lipid Res. 1996;37:299-308.

25. Li Z, Wang Y, van der Sluis RJ, van der Hoorn JW, Princen HM, Van Eck M, Van Berkel TJ, Rensen PC, Hoekstra M. Niacin reduces plasma cetp levels by diminishing liver macrophage content in cetp transgenic mice. Biochem Pharmacol. 2012;84:821-829.

26. Boon MR, Bakker LE, Haks MC, Quinten E, Schaart G, Van Beek L, Wang Y, Van Schinkel L, Van Harmelen V, Meinders AE, Ottenhoff TH, Van Dijk KW, Guigas B, Jazet IM, Rensen PC. Shortterm high-fat diet increases macrophage markers in skeletal muscle accompanied by impaired insulin signalling in healthy male subjects. Clin Sci (Lond). 2015;128:143-151.

27. Talbot CPJ, Plat J, Joris PJ, Konings M, Kusters Y, Schalkwijk CG, Ritsch A, Mensink RP. Hdl cholesterol efflux capacity and cholesteryl ester transfer are associated with body mass, but are not changed by diet-induced weight loss: A randomized trial in abdominally obese men. Atherosclerosis. 2018;274:23-28.

28. Verreck FA, de Boer T, Langenberg DM, van der Zanden L, Ottenhoff TH. Phenotypic and functional profiling of human proinflammatory type-1 and anti-inflammatory type-2 macrophages in response to microbial antigens and ifn-gamma- and cd40I-mediated costimulation. J Leukoc Biol. 2006;79:285-293.

29. Cipolletta C, Ryan KE, Hanna EV, Trimble ER. Activation of peripheral blood cd14+ monocytes occurs in diabetes. Diabetes. 2005;54:2779-2786.

30. Fadini GP, Cappellari R, Mazzucato M, Agostini C, Vigili de Kreutzenberg S, Avogaro A. Monocyte-macrophage polarization balance in pre-diabetic individuals. Acta Diabetol. 2013;50:977-982.

31. Yao Y, Xu XH, Jin L. Macrophage polarization in physiological and pathological pregnancy. Front Immunol. 2019;10:792.

32. Edwards SM, Cunningham SA, Dunlop AL, Corwin EJ. The maternal gut microbiome during pregnancy. MCN Am J Matern Child Nurs. 2017;42:310-317. 
33. Keuper M. On the role of macrophages in the control of adipocyte energy metabolism.

34. Greulich S, Maxhera B, Vandenplas G, de Wiza DH, Smiris K, Mueller H, Heinrichs J, Blumensatt $M$, Cuvelier C, Akhyari P, Ruige JB, Ouwens DM, Eckel J. Secretory products from epicardial adipose tissue of patients with type 2 diabetes mellitus induce cardiomyocyte dysfunction. Circulation. 2012;126:2324-2334.

35. Fernandez-Real JM, Perez del Pulgar S, Luche E, Moreno-Navarrete JM, Waget A, Serino M, Sorianello E, Sanchez-Pla A, Pontaque FC, Vendrell J, Chacon MR, Ricart W, Burcelin R, Zorzano A. Cd14 modulates inflammation-driven insulin resistance. Diabetes. 2011;60:21792186.

36. Jiang XC, Zhou HW. Plasma lipid transfer proteins. Curr Opin Lipidol. 2006;17:302-308.

37. Radeau T, Robb M, Lau P, Borthwick J, McPherson R. Relationship of adipose tissue cholesteryl ester transfer protein (cetp) mrna to plasma concentrations of cetp in man. Atherosclerosis. 1998;139:369-376.

38. Zhou H, Li Z, Hojjati MR, Jang D, Beyer TP, Cao G, Tall AR, Jiang XC. Adipose tissue-specific cetp expression in mice: Impact on plasma lipoprotein metabolism. J Lipid Res. 2006;47:20112019.

39. Dusserre E, Moulin P, Vidal H. Differences in mrna expression of the proteins secreted by the adipocytes in human subcutaneous and visceral adipose tissues. Biochim Biophys Acta. 2000;1500:88-96. 
Table 1. Characteristics of prediabetes $(n=20)$ vs. non-diabetes $(n=270)$ diagnosed at follow-up 5 years after the index pregnancy

\begin{tabular}{|c|c|c|c|c|c|c|c|c|c|c|}
\hline \multirow[t]{2}{*}{$\begin{array}{l}\text { Weeks of } \\
\text { pregnancy }\end{array}$} & \multicolumn{2}{|c|}{$14-16$} & \multicolumn{2}{|c|}{$22-24$} & \multicolumn{2}{|c|}{$30-32$} & \multicolumn{2}{|c|}{$36-38$} & \multicolumn{2}{|c|}{$\mathrm{FU}$} \\
\hline & Non-diabetes & Prediabetes & Non-diabetes & Prediabetes & Non-diabetes & Prediabetes & Non-diabetes & Prediabetes & Non-diabetes & Prediabetes \\
\hline $\mathrm{n}$ & 270 & 20 & 270 & 20 & 270 & 20 & 270 & 20 & 270 & 20 \\
\hline Age, year & $32.4(3.8)$ & $31.2(4.7)$ & & & & & & & $37.7(3.8)$ & $36.7(4.8)$ \\
\hline Height, cm & $169(6)$ & $170(6)$ & & & & & & & & \\
\hline BMI, $\mathrm{kg} / \mathrm{m}^{2}$ & $24(22,26)$ & $25(22,28)$ & $25(23,27)$ & 26. $(25,29)$ & $27(24,29)$ & $27(26,30)$ & $28(25,30$ & $28(26,31)$ & $23(21,25)$ & $26(24,30)^{* *}$ \\
\hline Weight, kg & $67(61,75)$ & $72(66,82)^{*}$ & $71(65,78)$ & $76(69,84)^{*}$ & $75(69,82)$ & $79(75,89)^{*}$ & $78(72,85)$ & $82(76,92)$ & $65(59,72)$ & $74(70,84)^{* * *}$ \\
\hline$\dagger$ Fat triceps & $19(15,24)$ & $21(15,27)$ & $20(15,25)$ & $21(17,27)$ & $20(15,25)$ & $20(19,29)$ & $19(15,24)$ & $19(17,26)$ & & \\
\hline †Fat subscapular & $16(13,22)$ & $22(14,30)^{*}$ & $18(13,25)$ & $22(17,37)^{*}$ & $19(14,26)$ & $22(18,39)^{*}$ & $19(15,28)$ & $25(14,38)$ & & \\
\hline †Fat iliaca & $23(16,32)$ & $31(22,35)^{*}$ & $27(21,34)$ & $31(24,39)$ & $28(22,35)$ & $35(24,40)$ & $28(22,35)$ & $33(25,37)$ & & \\
\hline SBP, mmHg & $110(100,120)$ & $110(110,120)$ & $110(100,115)$ & $113(100,120)$ & $110(105,120)$ & $110(110,130)$ & $110(105,120)$ & $110(110,130)$ & $110(100,120)$ & $120(106,130)$ \\
\hline DBP, mmHg & $70(60,70)$ & $70(66,74)^{*}$ & $65(60,70)$ & $70(65,79)^{*}$ & $70(60,70)$ & $70(66,79)^{*}$ & $70(65,80)$ & $70(70,75)$ & $70(60,75)$ & $70(69,75)$ \\
\hline Insulin, pmol/L & $26(18,37)$ & $37(30,57)^{* *}$ & $31(20,44)$ & $46(26,69)^{*}$ & $39(26,61)$ & $52(33,82)^{*}$ & $39(26,62)$ & $48(35,132)^{*}$ & $22(15,32)$ & $29(26,51)^{* *}$ \\
\hline Glucose, mmol/L & $4.6(4.3,4.8)$ & $4.9(4.7,5.2)^{* * * *}$ & & & $4.5(4.2,4.7)$ & $4.9(4.6,5.5)^{* * *}$ & $4.4(4.2,4.7)$ & $4.9(4.3,5.2)^{* *}$ & $4.7(4.4,5.0)$ & $5.6(5.6,5.9)^{* * * *}$ \\
\hline Insulin sensitivity & $210(149,296)$ & $140(87,223)^{* *}$ & & & $116(77,177)$ & $70(47,127)^{* *}$ & & & $250(178,339)$ & $145(70,195)^{* * *}$ \\
\hline Insulin resistance & $0.8(0.5,1.1)$ & $1.2(0.8,1.7)^{* *}$ & & & $1.1(0.7,1.8)$ & $1.5(1.0,3.0)^{* *}$ & $1.12(0.74,1.80)$ & $1.4(1.0,3.4)^{* *}$ & $0.7(0.4,1.0)$ & $1.1(0.9,1.7)^{* * * *}$ \\
\hline$\beta$-cell function & $1.2(0.9,1.5)$ & $0.8(0.7,0.8)^{* * * *}$ & & & $0.9(0.7,1.2)$ & $6.2(0.4,0.9)^{* * *}$ & & & $1.1(0.8,1.4)$ & $0.6(0.5,0.8)^{* * * *}$ \\
\hline
\end{tabular}


Table 2. Characteristics of GDM ( $\mathrm{n}=70)$ diagnosed with WHO 2013 criteria vs. non-GDM $(\mathrm{n}=215)$.

\begin{tabular}{|c|c|c|c|c|c|c|c|c|c|c|}
\hline & \multicolumn{2}{|c|}{$14-16$} & \multicolumn{2}{|c|}{$22-24$} & \multicolumn{2}{|c|}{$30-32$} & \multicolumn{2}{|c|}{$36-38$} & \multicolumn{2}{|r|}{$\mathrm{FU}$} \\
\hline & Non-GDM & GDM & Non-GDM & GDM & Non-GDM & GDM & Non-GDM & GDM & Non-GDM & GDM \\
\hline $\mathrm{n}$ & 215 & 70 & 215 & 70 & 215 & 70 & 215 & 70 & 215 & 70 \\
\hline Age, year & $32.0(3.7)$ & $33.0(4.1)^{*}$ & & & & & & & $37.4(3.7)$ & $38.4(4.2)^{*}$ \\
\hline Height, $\mathrm{cm}$ & $169(6)$ & $169(6)$ & & & & & & & & \\
\hline BMI, $\mathrm{kg} / \mathrm{m}^{2}$ & $24(21,25)$ & $25(23,27)^{* * *}$ & $25(23,27)$ & $27(24,28)^{* * *}$ & $26(24,28)$ & $28(26,30)^{* * *}$ & $27(25,30)$ & $29(26,31)^{* *}$ & $23(21,25)$ & $25(22,27)^{* * *}$ \\
\hline Weight, kg & $66(61,73)$ & $72(66,78)^{* * *}$ & $70(64,76)$ & $76(70,83)^{* * *}$ & $74(68,81)$ & $80(73,87)^{* * *}$ & $77(71,84)$ & $82(77,89)^{* * *}$ & $64(59,71)$ & $70(65,78)^{* * *}$ \\
\hline$\dagger$ Fat triceps & $19(15,23)$ & $21(16,26)^{*}$ & $19(15,24)$ & $21(18,26)^{* *}$ & $19(15,25)$ & $22(17,29)^{* *}$ & $19(14,23)$ & $20(17,29)^{* *}$ & & \\
\hline$\dagger$ Fat subscapular & $16(12,21)$ & $19(14,30)^{* *}$ & $18(13,24)$ & $22(16,34)^{* * *}$ & $18(14,25)$ & $21(17,33)^{* *}$ & $19(15,27)$ & $23(17,34)^{* *}$ & & \\
\hline$†$ Fat iliaca & $23(16,32)$ & $28(19,36)^{*}$ & $26(21,33)$ & $31(23,38)^{*}$ & $27(22,35)$ & $31(23,37)$ & $28(22,35)$ & $30(20,35)$ & & \\
\hline $\mathrm{SBP}, \mathrm{mmHg}$ & $110(100,119)$ & $110(110,120)^{* *}$ & $110(100,115)$ & $110(104,120)$ & $112(105,120)$ & $110(110,120)$ & $110(105,120)$ & $110(110,130)^{*}$ & $110(100,120)$ & $110(100,120)$ \\
\hline $\mathrm{DBP}, \mathrm{mmHg}$ & $70(60,70)$ & $70(60,70)$ & $65(60,70)$ & $65(60,70)$ & $70(60,70)$ & $70(60,70)$ & $70(69,80)$ & $70(65,80)$ & $70(60,75)$ & $70(64,75)$ \\
\hline Insulin, $\mathrm{pmol} / \mathrm{L}$ & $25(16,36)$ & $33(26,50)^{* * *}$ & $28(19,41)$ & $37(26,58)^{* * *}$ & $35(24,53)$ & $53(39,76)^{* * *}$ & $36(25,61)$ & $45(34,76)^{* *}$ & $21(15,31)$ & $29(20,40)^{* * *}$ \\
\hline Glucose, $\mathrm{mmol} / \mathrm{L}$ & $4.5(4.3,4.7)$ & $5.0(4.6,5.2)^{* * *}$ & & & $4.4(4.2,4.6)$ & $5.0(4.6,5.3)^{* * *}$ & $4.4(4.2,4.6)$ & $4.9(4.6,5.2)^{* * *}$ & $4.7(4.4,5.0)$ & $5.0(4.7,5.3)^{* * *}$ \\
\hline Insulin sensitivity & $223(159,314)$ & $152(104,200)^{* * *}$ & & & $123(86,182)$ & $76(51,121)^{* * *}$ & & & $256(191,349)$ & $181(124,270)^{* * *}$ \\
\hline Insulin resistance & $0.70(0.46,1.04)$ & $0.99(0.81,1.61)^{* * *}$ & & & $1.0(0.7,4.5)$ & $1.7(1.1,2.6)^{* * *}$ & $1.0(0.7,1.7)$ & $1.4(1.0,2.4)^{* * *}$ & $0.6(0.4,0.9)$ & $0.9(0.6,1.3)^{* * *}$ \\
\hline$\beta$-cell function & $1.2(1.0,1.6)$ & $0.8(0.6,1.1)^{* * *}$ & & & $1.0(0.8,1.3)$ & $0.6(0.4,0.7)^{* * *}$ & & & $1.1(0.9,1.4)$ & $0.8(0.6,1.2)^{* * *}$ \\
\hline
\end{tabular}


Table 3. Plasma levels of adipokines, monocyte/macrophage and inflammation markers in non-diabetes vs. prediabetes

\begin{tabular}{|c|c|c|c|c|c|c|c|c|c|c|}
\hline & \multicolumn{2}{|c|}{$14-16$} & \multicolumn{2}{|c|}{$22-24$} & \multicolumn{2}{|c|}{$30-32$} & \multicolumn{2}{|c|}{$36-38$} & \multicolumn{2}{|c|}{ FU } \\
\hline & Non-diabetes & Prediabetes & Non-diabetes & Prediabetes & Non-diabetes & Prediabetes & Non-diabetes & Prediabetes & Non-diabetes & Prediabetes \\
\hline \multicolumn{11}{|l|}{ Adipokines } \\
\hline Adiponectin & $8.5(8.1-8.9)$ & $7.5(6.3-8.9)$ & $7.8(7.4-8.2)$ & $6.3(5.2-7.7)$ & $7.0(6.6-7.3)$ & $7.2(5.9-8.8)$ & $7.1(6.8-7.5)$ & $6.9(5.8-8.2)$ & $7.8(7.4-8.2)$ & $6.3(5.2-7.6)$ \\
\hline Leptin & $43(41-45)$ & $44(37-53)$ & $47(45-49)$ & $46(39-56)$ & $52(49-55)$ & $54(44-67)$ & $50(47-53)$ & $52(41-65)$ & $27(25-28)$ & $32(25-40)$ \\
\hline Resistin & $35(33-36)$ & $40(34-47)$ & $37(36-39)$ & $42(35-49)$ & $38(36-40)$ & $34(28-42)$ & $42(40-44)$ & $43(36-51)$ & $27(26-29)$ & $32(27-38)$ \\
\hline Chemerin & $198(192-204)$ & $206(184-231)$ & $212(206-219)$ & $202(179-227)$ & $220(213-228)$ & $234(206-267)$ & $239(231-247)$ & $230(202-260)$ & $175(170-181)$ & $200(177-226)$ \\
\hline \multicolumn{11}{|c|}{ Monocyte/macrophage markers } \\
\hline sCD163 & $533(508-558)$ & $545(457-651)$ & $681(648-717)$ & $624(517-754)$ & $734(696-775)$ & $718(586-878)$ & $792(754-831)$ & $742(619-890)$ & $503(479-528)$ & $515(430-618)$ \\
\hline sCD14 & $2.7(2.6-2.8)$ & $3.1(2.6-3.6)$ & $2.8(2.7-2.9)$ & $2.8(2.4-3.2)$ & $3.1(2.9-3.2)$ & $3.5(3.0-4.1)$ & $3.2(3.1-3.3)$ & $3.4(3.0-3.9)$ & $3.2(3.1-3.3)$ & $3.5(3.1-4.0)$ \\
\hline \multicolumn{11}{|c|}{ Inflammatory markers } \\
\hline CRP & $1.5(1.4-1.6)$ & $1.9(1.4-2.7)$ & $1.5(1.4-1.7)$ & $2.2(1.6-3.0)$ & $1.3(1.2-1.4)$ & $1.7(1.2-2.4)$ & $1.3(1.2-1.4)$ & $1.5(1.0-2.1)$ & $0.4(0.3-0.4)$ & $0.6(0.4-1.0)$ \\
\hline
\end{tabular}

544 Data are given as back-transformed (from log values) estimated marginal means and 96\% confidence intervals. 
Table 4. CETP activity associated with clinical variables, lipoproteins, adipokines, monocyte/macrophage and inflammatory markers during pregnancy and follow-up ( $\mathrm{n}=290$ )

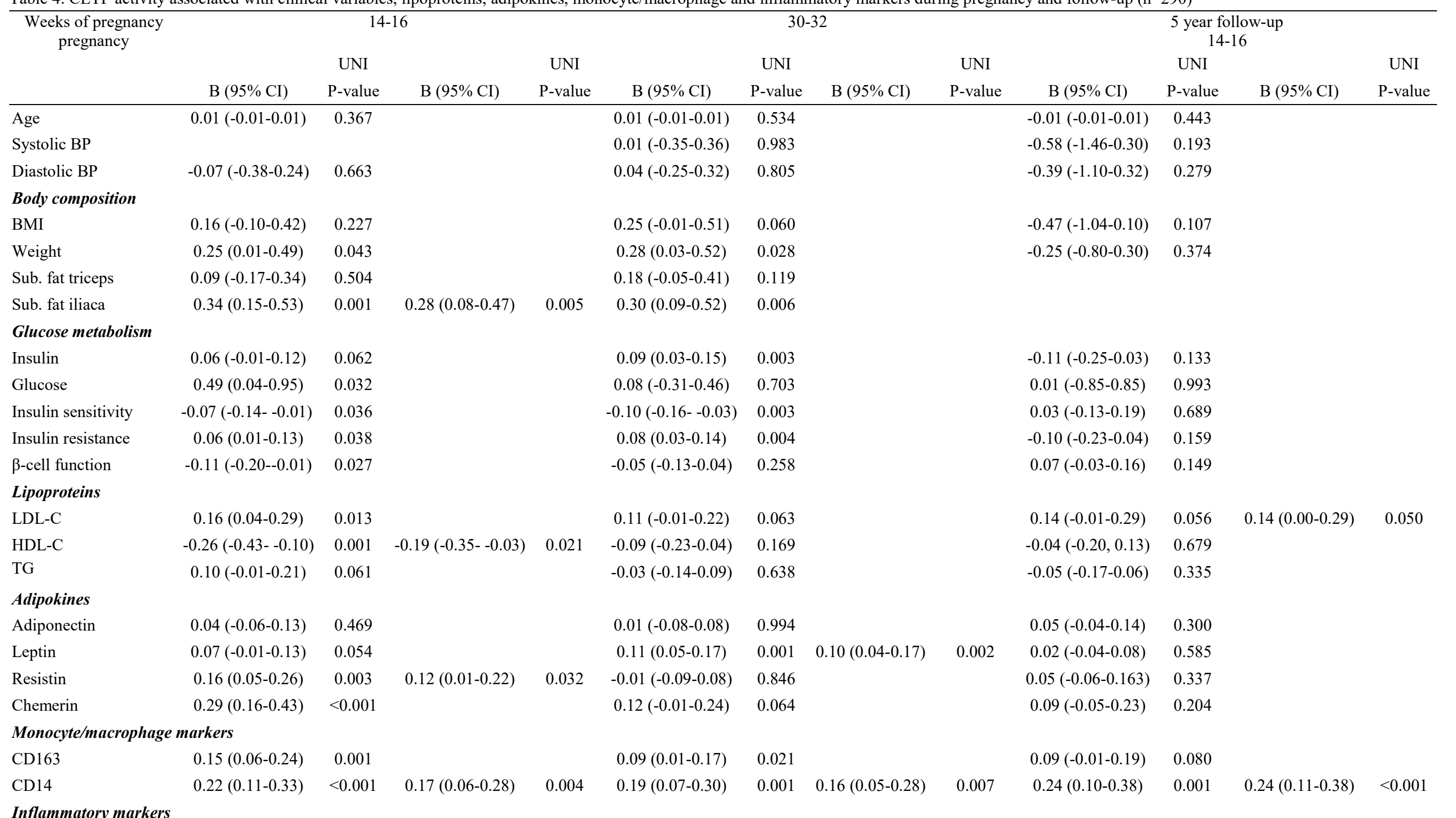


\#The association of the lipoprotein data and CETP activity is from week 36-38 instead of 30-32 week. Numbers are correlation coefficient, slope (beta) and p-values. 
Table 5. Uni and multivariable logistic and linear regression identifying the strongest predictors of prediabetes and $2 \mathrm{~h}$ glucose during OGTT after 5 years follow-up

\begin{tabular}{|c|c|c|c|c|c|c|c|c|c|c|}
\hline & \multicolumn{6}{|c|}{ Prediabetes at 5 years follow-up } & \multicolumn{4}{|c|}{$2 \mathrm{~h}$ glucose 5 years after pregnancy } \\
\hline & \multicolumn{3}{|c|}{ Univariate } & \multicolumn{3}{|c|}{ Multivariable } & \multicolumn{2}{|c|}{ Univariate } & \multicolumn{2}{|l|}{ Multivariable } \\
\hline & Slope & $\mathrm{RR}(95 \% \mathrm{CI})$ & $\mathrm{P}$ & Slope & $\mathrm{RR}(95 \% \mathrm{CI})$ & $\mathrm{P}$ & Slope $(95 \%$ CI $)$ & $\mathrm{P}$ & Slope $(95 \%$ CI $)$ & $\mathrm{P}$ \\
\hline Age & -0.08 & $0.92(0.82-1.04)$ & 0.177 & -0.11 & $0.90(0.78-1.03)$ & 0.129 & $0.00(-0.04-0.03)$ & 0.77 & & \\
\hline BMI & 0.39 & $1.48(0.97-2.27)$ & 0.070 & -0.05 & $0.95(0.52-1.74)$ & 0.901 & $0.31(0.20-0.42)$ & $<0.001$ & $0.10(-0.08-0.27)$ & 0.27 \\
\hline Diabetes in family & 0.53 & $1.69(0.71-4.05)$ & 0.24 & & & & $0.16(-0.08-0.40)$ & 0.20 & $0.08(-0.15-0.32)$ & 0.48 \\
\hline Parity & 0.56 & $1.75(0.67-4.59)$ & 0.25 & & & & $0.12(-0.12-0.36)$ & 0.32 & & \\
\hline LDL-C & 0.11 & $1.11(0.70-1.76)$ & 0.65 & & & & $0.09(-0.02-0.21)$ & 0.117 & $0.02(-0.09-0.13)$ & 0.73 \\
\hline HDL-C & -0.55 & $0.58(0.26-0.92)$ & 0.021 & -0.05 & $0.95(0.52-1.74)$ & 0.87 & $-0.26(-0.37--0.15)$ & $<0.001$ & $-0.09(-0.21-0.03)$ & 0.157 \\
\hline$\beta$-cell function & -1.10 & $0.33(0.20-0.57)$ & $<0.001$ & -1.05 & $0.35(0.19-0.66)$ & 0.001 & $-0.34(-0.45--0.22)$ & $<0.001$ & $-0.22(-0.34--0.10)$ & $<0.001$ \\
\hline CETP & 0.68 & $1.97(1.20-3.24)$ & $<0.001$ & 0.75 & $2.13(1.18-3.81)$ & 0.012 & $0.22(0.11-0.34)$ & $<0.001$ & $0.15(0.03-0.27)$ & 0.012 \\
\hline Resistin & 0.33 & $1.40(0.89-2.20)$ & 0.149 & 0.30 & $1.35(0.76-2.39)$ & 0.30 & $0.12(0.00-0.24)$ & 0.045 & $0.10(-0.02-0.21)$ & 0.099 \\
\hline Chemerin & 0.23 & $1.26(0.80-1.98)$ & 0.33 & & & & $0.19(0.07-0.30)$ & 0.002 & $-0.08(-0.22-0.06)$ & 0.28 \\
\hline Leptin & 0.40 & $1.49(0.92-2.42)$ & 0.106 & 0.15 & $1.16(0.52-2.58)$ & 0.72 & $0.31(0.19-0.42)$ & $<0.001$ & $0.16(-0.01-0.21)$ & 0.064 \\
\hline sCD163 & 0.13 & $1.14(0.72-1.80)$ & 0.59 & & & & $0.21(0.09-0.32)$ & 0.001 & $0.07(-0.06-0.20)$ & 0.30 \\
\hline sCD14 & 0.27 & $1.31(0.81-2.11)$ & 0.27 & & & & $0.11(0.00-0.23)$ & 0.062 & $0.06(-0.08-0.19)$ & 0.41 \\
\hline
\end{tabular}


Table 6. Influence of covariates on the association between CETP at 14-16 weeks and

prediabetes and $2 \mathrm{~h}$ glucose 5 years after pregnancy.

\begin{tabular}{|c|c|c|c|c|c|}
\hline & \multicolumn{3}{|c|}{ Prediabetes at 5 years follow-up } & \multicolumn{2}{|c|}{$2 \mathrm{~h}$ glucose 5 years after pregnancy } \\
\hline & Slope & $\mathrm{RR}(95 \% \mathrm{CI})$ & $\mathrm{P}$ & & \\
\hline CETP Unadjusted & 0.68 & $1.97(1.20-3.24)$ & 0.007 & $0.23(0.11-0.34)$ & $<0.001$ \\
\hline Adjusted by: & & & & & \\
\hline Age & 0.70 & $2.01(1.22-3.31)$ & 0.006 & $0.23(0.11-0.35)$ & $<0.001$ \\
\hline BMI & 0.79 & $2.19(1.29-3.73)$ & 0.004 & $0.22(0.11-0.33)$ & $<0.001$ \\
\hline Diabetes in family & 0.70 & $2.00(1.22-3.30)$ & 0.006 & $0.23(0.11-0.35)$ & $<0.001$ \\
\hline Parity & 0.72 & $2.06(1.23-3.45)$ & 0.006 & $0.23(0.11-0.35)$ & $<0.001$ \\
\hline LDL-C & 0.68 & $1.97(1.19-3.24)$ & 0.008 & $0.22(0.10-0.34)$ & $<0.001$ \\
\hline HDL-C & 0.63 & $1.87(1.13-3.10)$ & 0.015 & $0.19(0.07-0.30)$ & 0.001 \\
\hline$\beta$-cell function & 0.62 & $1.85(1.10-3.12)$ & 0.021 & $0.19(0.07-0.31)$ & 0.002 \\
\hline Resistin & 0.67 & $1.95(1.17-3.24)$ & 0.010 & $0.21(0.10-0.33)$ & $<0.001$ \\
\hline Chemerin & 0.66 & $1.94(1.16-3.23)$ & 0.011 & $0.19(0.07-0.31)$ & 0.002 \\
\hline Leptin & 0.67 & $1.96(1.18-3.26)$ & 0.010 & $0.20(0.08-0.31)$ & 0.001 \\
\hline sCD163 & 0.68 & $1.97(1.19-3.24)$ & 0.008 & $0.20(0.08-0.31)$ & 0.001 \\
\hline sCD14 & 0.65 & $1.92(1.16-3.18)$ & 0.012 & $0.21(0.09-0.33)$ & $<0.001$ \\
\hline
\end{tabular}

BMI, body mass index; CETP, cholesteryl ester transfer protein; LDL-C, low-density lipoprotein cholesterol; HDL-C, high-density lipoprotein cholesterol; RR, relative risk. 
Figure 1. Lipoproteins and lipids during pregnancy and 5 years follow-up in prediabetes, GDM and

597 control women. In the figure GDM is present both in the GDM group and in the control group.

598 Statistics is performed comparing prediabetes vs. non-diabetes $\left(^{*}\right)$ and GDM vs. non-GDM ( $\left.\dagger\right)$ at each

599 timepoints. $\dagger^{*} p<0.05 \dagger^{* * *} \mathrm{p}<0.01, \uparrow \dagger \uparrow \mathrm{p}<0.001$

600
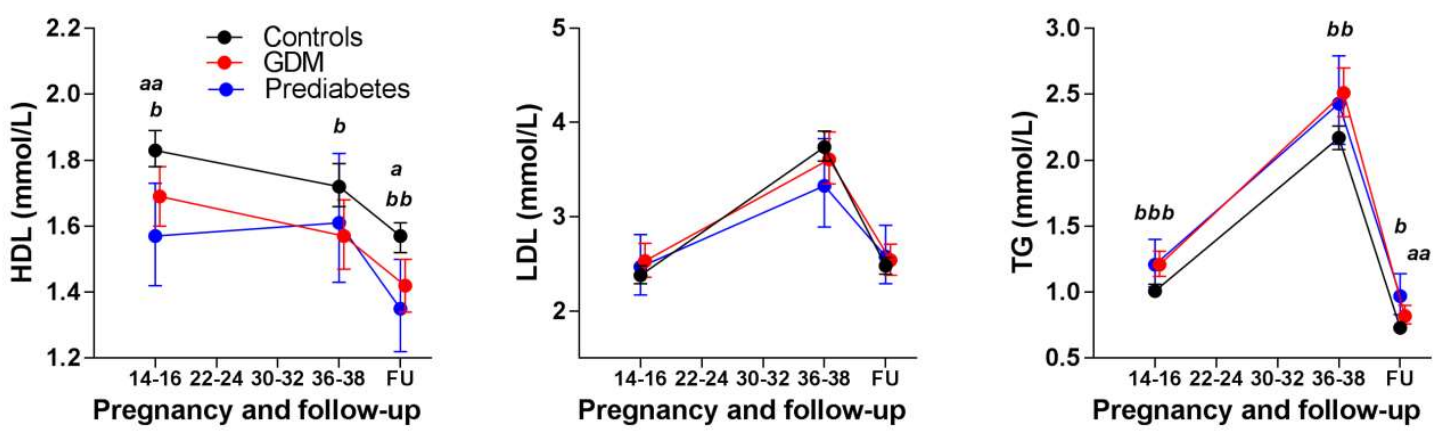

601

602

603

604

605

606

607

608

609

610

611

612

613

614

615

616 
617 Figure 2. CETP activity during pregnancy and 5 years follow-up in GDM, prediabetes and control

618 women. $* \mathrm{p}<0.05 * * \mathrm{p}<0.01$ between non-diabetes vs. pre-diabetes. P-values denote group effect from 619 the repeated measures ANOVA.

620

621

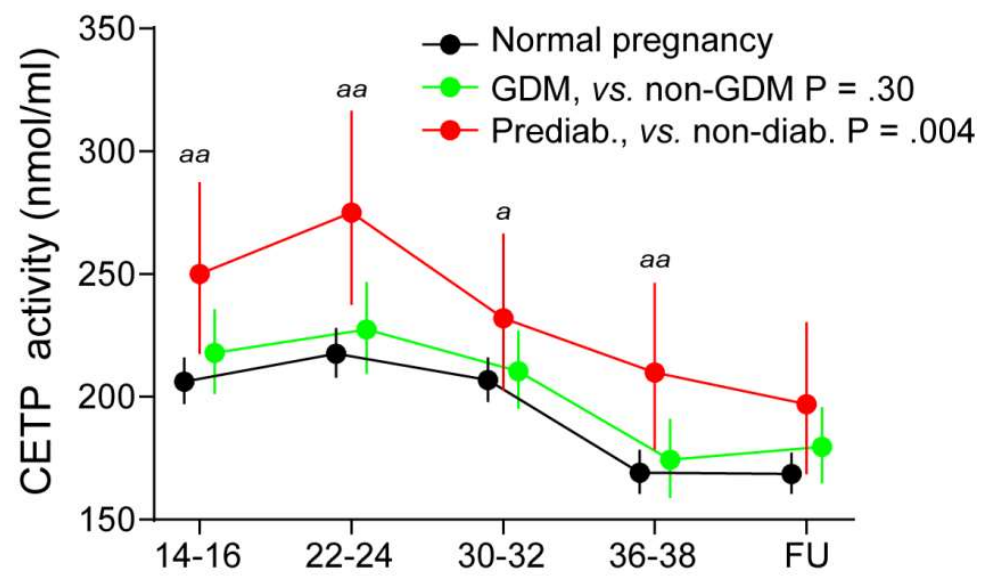

623

624

625

626

627

628

629

630

631

632

633

634 
Figure 3. Association between CETP and sCD14 during pregnancy. Circulating CETP activity and

636

637

638

639

640

641

642

643

644

645 sCD14 levels at A. week 14-16 and B. expressed as area under the curve (AUC) for both markers during normal pregnancy, in GDM and in women with prediabetes at 5 years follow-up. ${ }^{* *} \mathrm{p}<0.01 v s$. Normal pregnancy in A/B. C. correlation between AUC's for sCD14 and CETP. Correlation coefficients and p-values are from Spearman correlation. D. Associations (Spearman) between CETP and sCD14 at different time-points during pregnancy in normal pregnancy, prediabetes and GDM. E. Interaction analysis of 2-hour glucose levels at 5-year follow-up. F. product of CETP and sCD14 tertiles (i.e. CETP tertile*sCD14 tertile) which gives 6 groups: group 1: Tertile 1 (T1) of both CETP and SCD14; group 2: combinations of T1 and T2 of CETP and SCD14; group 3: combinations of T1 and T3 of CETP and sCD14; group 4: T2 of both; group 5: combinations of T2 and T3 of CETP and $\mathrm{sCD} 14$; group 6: T3 of both. ${ }^{* *} \mathrm{p}<0.01$ vs. group 1-3.
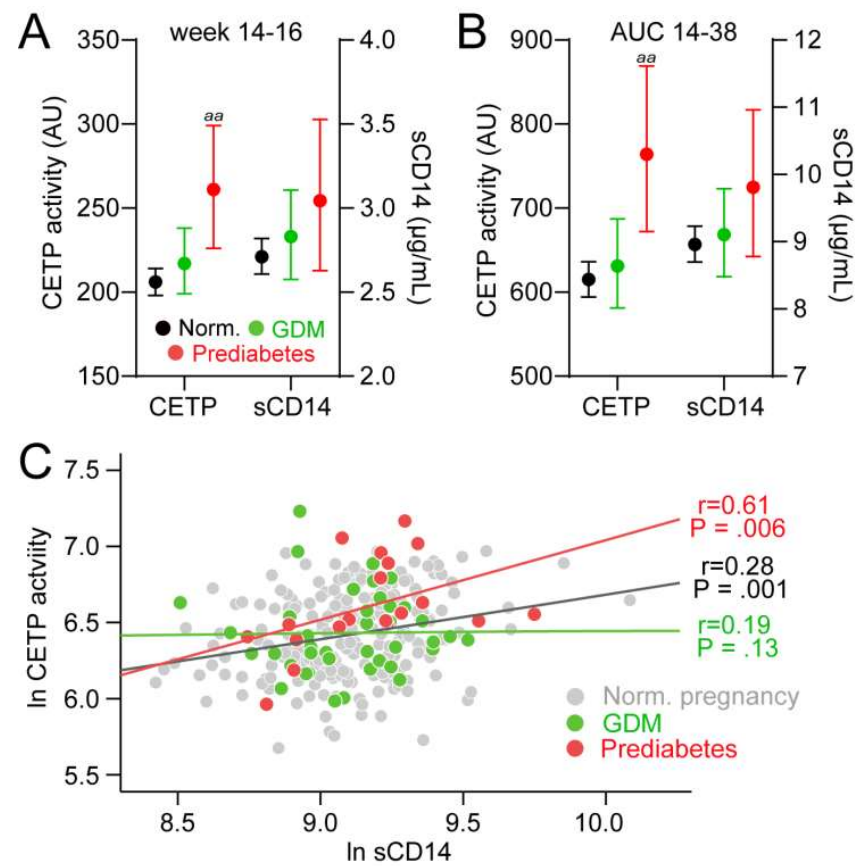

\begin{tabular}{ccccc}
$\mathrm{D}$ & \multicolumn{1}{c}{$14-16$} & \multicolumn{1}{c}{$22-24$} & \multicolumn{1}{c}{$30-32$} & \multicolumn{1}{c}{$36-38$} \\
\hline Norm. preg. & $0.23(.001)$ & $0.22(.001)$ & $0.24(<.001)$ & $0.28(<.001)$ \\
GDM & $0.24(.046)$ & $0.18(.14)$ & $0.21(.077)$ & $0.07(.59)$ \\
Prediabetes & $0.39(.087)$ & $0.46(.041)$ & $0.55(.012)$ & $0.41(.084)$
\end{tabular}

\section{$\mathrm{E}$ \begin{tabular}{lrrr} 
Parameter & Slope & $95 \% \mathrm{Cl}$ & Sig. \\
\hline Intercept & -0.03 & -0.15 to 0.08 & .581 \\
CETP* & 0.13 & 0.02 to 0.25 & .025 \\
SCD14 & 0.10 & -0.02 to 0.22 & .117 \\
sCD14 & 0.10 & 0.10 to 0.33 & .001
\end{tabular}}

646

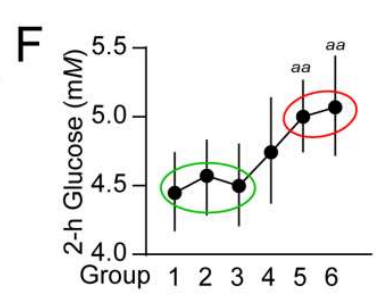


